ARTICLE

DOI: $10.1038 / s 41467-018-03073-7$

\title{
Cytosine modifications exhibit circadian oscillations that are involved in epigenetic diversity and aging
}

Gabriel Oh${ }^{1}$, Sasha Ebrahimi ${ }^{1}$, Matthew Carlucci ${ }^{1}$, Aiping Zhang ${ }^{1}$, Akhil Nair ${ }^{1}$, Daniel E. Groot ${ }^{1}$, Viviane Labrie ${ }^{1,2}$, Peixin Jia', Edward S. Oh, Richie H. Jeremian ${ }^{1}$, Miki Susic ${ }^{1}$, Tenjin C. Shrestha ${ }^{3,4}$, Martin R. Ralph ${ }^{4}$, Juozas Gordevičius ${ }^{5,6}$, Karolis Koncevičius ${ }^{5,6}$ \& Art Petronis ${ }^{1,5}$

Circadian rhythmicity governs a remarkable array of fundamental biological functions and is mediated by cyclical transcriptomic and proteomic activities. Epigenetic factors are also involved in this circadian machinery; however, despite extensive efforts, detection and characterization of circadian cytosine modifications at the nucleotide level have remained elusive. In this study, we report that a large proportion of epigenetically variable cytosines show a circadian pattern in their modification status in mice. Importantly, the cytosines with circadian epigenetic oscillations significantly overlap with the cytosines exhibiting age-related changes in their modification status. Our findings suggest that evolutionary advantageous processes such as circadian rhythmicity can also contribute to an organism's deterioration.

\footnotetext{
${ }^{1}$ The Krembil Family Epigenetics Laboratory, The Campbell Family Mental Health Research Institute, Centre for Addiction and Mental Health, Toronto, ON M5T 1R8, Canada. ${ }^{2}$ Center for Neurodegenerative Science, Van Andel Research Institute, Grand Rapids, MI 49503, USA. ${ }^{3}$ Department of Cell and Systems Biology, University of Toronto, Toronto, ON M5S 3G5, Canada. ${ }^{4}$ Department of Psychology, University of Toronto, Toronto, ON M5S 3G5, Canada.

${ }^{5}$ Institute of Biotechnology, Life Sciences Center, Vilnius University, Vilnius, LT-10257, Lithuania. ${ }^{6}$ Institute of Data Science and Digital Technologies, Vilnius University, Vilnius, LT-08663, Lithuania. Gabriel Oh and Sasha Ebrahimi contributed equally to this work. Correspondence and requests for materials should be addressed to A.P. (email: Art.Petronis@camh.ca)
} 
C ircadian rhythmicity is one of the oldest evolutionary adaptations to day and night cycles. It regulates a wide spectrum of biological phenomena, from temperaturedependent fluctuations in biochemical reaction rates of prokaryotes, to sleep-wake cycles and higher-order behaviors in multicellular organisms ${ }^{1}$. Disruptions of circadian rhythms have been linked to human morbidities, including cancer, mood, and neurodegenerative diseases ${ }^{2,3}$. Relatedly, numerous studies have shown an association between circadian disruption and aging. For instance, in older rodents, circadian regulation becomes weaker, whereas mice deficient in key circadian genes have shorter lifespans ${ }^{4}$. The cause-effect relationship and molecular mechanisms of this association are yet to be uncovered.

The cell-autonomous circadian clock consists of a series of transcription factors and regulators that coordinate feedback loops. In mammals, the clock circadian regulator (CLOCK) transcription factor forms a heterodimer with the aryl hydrocarbon receptor nuclear translocator-like protein (ARNTL, also known as BMAL1). This complex binds to the E-box response elements to regulate expression of clock controlled genes ${ }^{5,6}$. This set of activated genes includes Period (PER1, 2, and 3) and Cryptochrome (CRY1 and CRY2), which initiate a negative feedback in this pathway. Circadian-regulated genes are ubiquitous and partially tissue specific: up to $55 \%$ of protein-coding genes exhibit circadian transcriptional oscillations in at least 1 of 12 mouse tissues ${ }^{7}$. A number of post-translational histone modifiers also mediate circadian regulation. CLOCK, for instance, functions as an acetyltransferase of histone $\mathrm{H} 3$ at $\mathrm{K} 9$ and $\mathrm{K} 14$ positions and interacts with other histone acetyltransferases ${ }^{8}$. Similarly, other circadian factors, directly or through formation of complexes with other enzymes, control histone deacetylation ${ }^{9}$ and methylation ${ }^{10}$. Genome-wide chromatin immunoprecipitation experiments have demonstrated coordinated circadian oscillations of histone $\mathrm{H} 3 \mathrm{~K} 4$ trimethylation and $\mathrm{H} 3 \mathrm{~K} 9 / \mathrm{H} 3 \mathrm{~K} 27$ acetylation at transcription start sites of expressed genes, as well as $\mathrm{H} 3 \mathrm{~K} 4$ monomethylation and H3K27 acetylation at enhancers ${ }^{11}$. Contrary to the strong evidence for histone oscillations, the role of cytosine modification, mainly comprised of 5-methyl- and 5-hydroxymethylcytosines (hmCs), in the maintenance or modulation of the circadian clock is unclear. Even though cyclical changes with $24 \mathrm{~h}$ periodicity have been demonstrated in key elements of the cytosine modification machinery (such as DNA methyltransferases, Ten-eleven translocation enzymes, and global DNA methylation levels ${ }^{12}$ ), dedicated studies have previously failed to detect evidence for robust $24 \mathrm{~h}$ periodicity of cytosine modification patterns in mice $^{13,14}$. In humans, a study of postmortem brain samples revealed vestiges of circadian oscillations, which accounted for a small fraction $(<0.3 \%)$ of the total inter-individual cytosine modification variance ${ }^{15}$.

In this study (Fig. 1), we demonstrate that cytosine modification profiles are changing in a circadian manner in the mouse liver and lung. Oscillating modified cytosines (osc-modCs) are more prevalent in both high expressing and circadian genes and were enriched for E-box motifs. We also find that osc-modCs were associated with the aging epigenome, where the amplitude of the oscillation correlated with the magnitude of the aging effect, implying common molecular mechanisms and shedding a new light on the proximal causes of aging.

\section{Results}

Circadian oscillations of modCs. We investigated circadian oscillations of cytosine modification in 9-, 15-, and 25-month-old (mo) male mice ( $n=36,30$, and 30 , respectively) to represent an aging spectrum from adult to the very old. All mice were individually housed, with ad libitum access to food and water, and entrained on a $12 \mathrm{~h}$ light : $12 \mathrm{~h}$ dark cycle, where Zeitgeber time (ZT) 0 is light onset and ZT12 is light offset. The circadian entrainment of mice was verified using locomotor activity and messenger RNA profiles of two key circadian genes, Per2 and Arntl $^{1}$ (Fig. 2a-f). Liver and lung tissues, which exhibit robust transcriptional oscillations and are frequently used in circadian studies $^{7}$, were collected every $2 \mathrm{~h}$ for at least $58 \mathrm{~h}$ starting at ZT0.

We targeted chromosome 7, which is relatively small and gene dense, to gain sufficient depth of sequencing. In order to gain precise measurements of oscillating cytosine modifications all experiments were performed in technical triplicates, and the cytosine modification measurements were filtered for sites that showed a greater degree of biological variation than technical variation (henceforth referred to as epigenetically variable cytosines (EVCs); see Methods for more details). We performed mapping of osc-modCs at the single nucleotide resolution using 10,696 bisulfite padlock probes ${ }^{16}$, each targeting a unique 130 $140 \mathrm{bp}$ region of chr 7, followed by sequencing to an average mapped read depth of $\sim 1,800 \times$ per CpG (Supplementary Fig. 1). Out of the 37,249 targeted CpGs (Supplementary Data 1), we

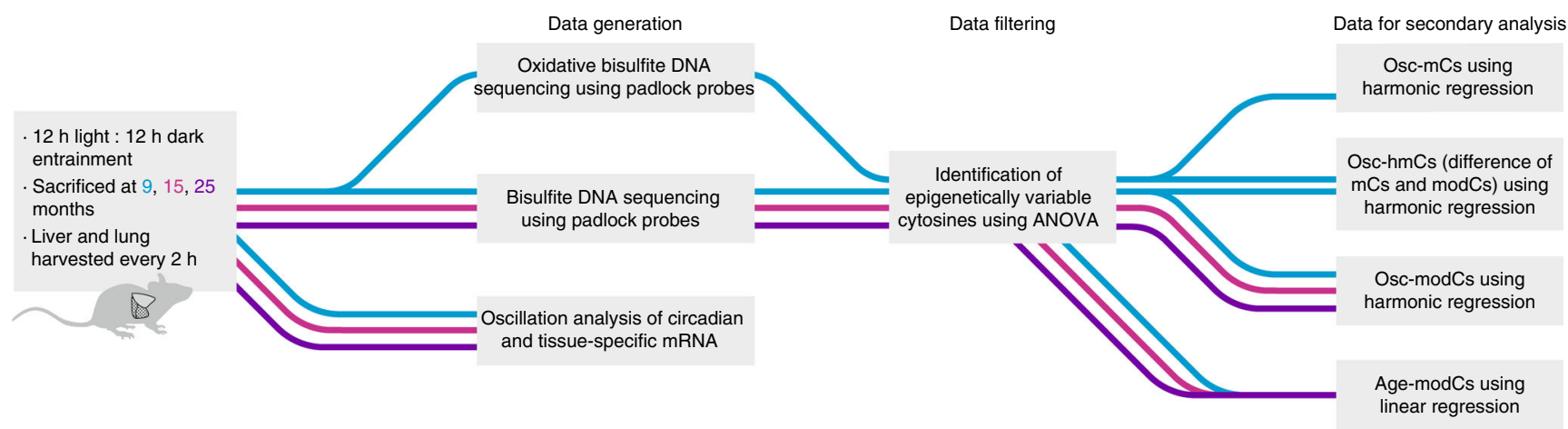

Fig. 1 Experimental workflow summary. We investigated 9-, 15-, and 25-month-old (mo) male mice entrained on a $12 \mathrm{~h}$ light : $12 \mathrm{~h}$ dark cycle with ad libitum access to food. The circadian entrainment of mice was verified using locomotor activity and mRNA profiles of key circadian genes. The confounding effects of cellular heterogeneity were examined using cell-specific non-circadian mRNAs. All sequencing experiments were performed in technical triplicates. ANOVA was used to select for cytosines whose variation of modification densities were larger in the biological samples compared with technical replicates, which we refer to as epigenetically variable cytosines (EVCs). 5-Hydroxymethylcytosine densities were estimated by subtracting mC from modC densities on CpG sites that are intersected across EVCs. Aging and oscillating cytosines were identified using linear and harmonic regression models, respectively. Age-modC, age-correlated cytosine modifications; Osc-hmC, oscillating 5-hydroxymethylcytosines; Osc-mC, oscillating 5-methylcytosines; Osc-modC, oscillating modified cytosines 

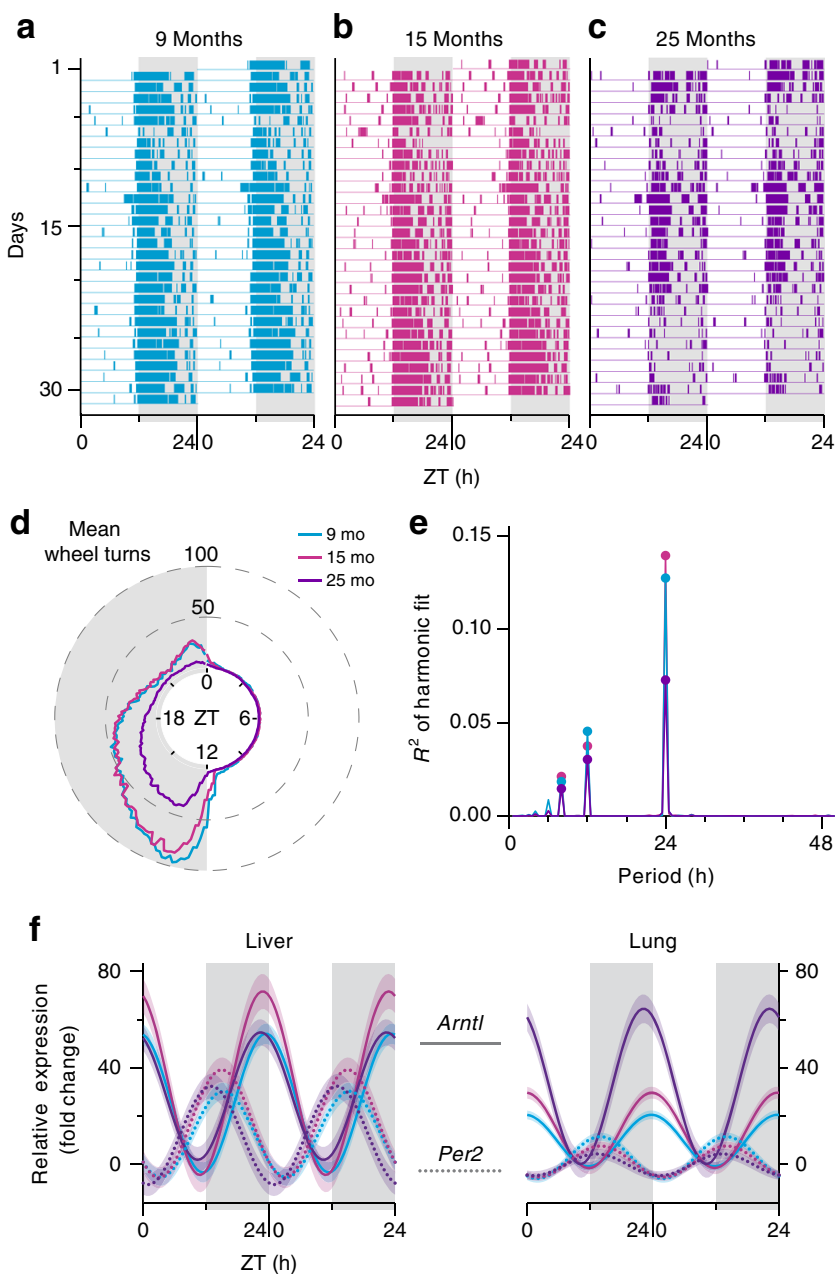

Fig. 2 Locomotor activity and molecular markers verifying circadian entrainment of mice. a-c Representative actograms of individual mice from a 9-mo, b 15-mo, and c 25-mo cohorts. The colored bars represent wheel running activity over 30 days. The light and grey columns show periods of lights on and off. By convention, each line depicts 2 days of activity, with the second day appearing again in the line below (i.e., double-plotted). d The summarized activity of each age cohort measured as the mean number of turns for each mouse in the age group (measured in 6 min intervals over 30 days) as a function of ZT. e Spectral analysis of the actograms in each age cohort showing the $R^{2}$ of harmonic fits at varying periodicities $(1 \mathrm{~h}$ intervals). $\mathbf{f}$ Twenty-four hours harmonic regression fits of relative mRNA levels of two antiphasic circadian genes, Arntl and Per2, in liver and lung tissues of all three age cohorts. The data are double plotted by convention. Shading around the regression lines represents the 95\% confidence band. ZT, Zeitgeber time; mo, month-old

successfully captured $\sim 28,000$ CpGs, of which $\sim 13,000$ were epigenetically variable.

We plotted the average modification density within $1 \mathrm{Mb}$ windows of chromosome 7 and observed a synchronized pattern of oscillation in the lung samples, whereas a less organized pattern was detected in the liver. Harmonic regression analysis, which can identify sinusoidal patterns with $24 \mathrm{~h}$ periodicity, was used to determine the periodic relationship between $\mathrm{ZT}$ and modification levels. It showed that chromosome-wide average modification oscillated with a $24 \mathrm{~h}$ periodicity in the lung $(p=$ $2.9 \times 10^{-7}$ ) but not in the liver $(p=0.74)$ (Fig. 3a-b). Principal component analysis (PCA) revealed that the oscillating first principal component (harmonic regression $p=1.8 \times 10^{-6}$ ) explained $25 \%$ of the cytosine modification variance in the lung, whereas PCA in the liver interestingly revealed oscillating principal components 2 and 3 (harmonic regression $p=0.028$ and 0.015 , respectively), which cumulatively explained $13 \%$ of the variance (Fig. 3c-d). Analysis of individual CpGs revealed oscmodCs in $8.2 \%$ (permuted $p=0.046$ ) and $35.6 \%$ (permuted $p<10^{-4}$ ) of EVCs in the liver and lung, respectively (Fig. 4a-f, Supplementary Fig. 2, and Supplementary Data 2-3). The mean amplitudes of oscillation were $3.2 \pm 1.8 \%$ (mean \pm SD) and $4.5 \pm$ $2.2 \%$, with maximum amplitudes of $14 \%$ and $17 \%$, in the liver and lung, respectively. Some osc-modCs were not tissue specific, as genomic positions of osc-modCs significantly overlapped between the liver and lung (odds ratio (OR) (95\% confidence interval $\left.)=2.0(1.7-2.4) ; p=1.2 \times 10^{-20}\right)$, and shared a similar acrophase (ZT when oscillation reaches its peak) with a median absolute acrophase difference of $2.1 \mathrm{~h}$ (permuted $p<1.0 \times 10^{-4}$; Supplementary Fig. 3).

Consistent with the general trend of diminishing circadian effects with age $\mathrm{e}^{3,4}$, we observed a decreasing proportion of oscmodCs in the older animals. Compared with the 9-mo cohort, lung osc-modCs were reduced slightly in the 15 -mo mice $(28.1 \%)$ and dropped more dramatically in the 25 -mo mice $(13.9 \%$; Supplementary Fig. 4 and Supplementary Data 4-5), and these effects were not influenced by differences in cohort sample sizes (Supplementary Table 1). Liver did not exhibit significant oscillations in the older age cohorts (Supplementary Fig. 4 and Supplementary Data 6-7). As only 9-mo mice showed consistently significant oscillations in both tissues, we focused primarily on this group for all subsequent circadian cytosine modification analyses.

Mechanistically, genuine osc-modCs must involve DNA demethylation, probably by the oxidation of 5-methylcytosine (mC) to $\mathrm{hmC}^{17}$, followed by remethylation. Using oxidative bisulfite (oxBS) conversion $^{18}$ followed by sequencing using padlock probes, we detected mostly significant oscillations in both hmC and $\mathrm{mC}$ densities (permuted $p=0.011$ and 0.095 , for liver; $p=0.012$ and $7.8 \times 10^{-3}$, for lung; Supplementary Fig. 5a-1 and Supplementary Data 8-11). Although both types of oscillating modifications overlapped $(\mathrm{OR}=6.3(5.2-7.6)$ and $10.2(5.7-18.4)$; $p=9.2 \times 10^{-71}$ and $7.4 \times 10^{-16}$ in the liver and lung, respectively), their respective acrophases were in antiphase with one another (11.2 and $11.0 \mathrm{~h}$ median absolute acrophase difference between the two; permuted $p<1 \times 10^{-4}$ and $5.4 \times 10^{-3}$ in the liver and lung, respectively; Supplementary Fig. $5 \mathrm{~m}-\mathrm{p}$ ). This indicates coordinated timing of DNA demethylation and remethylation during the circadian cycle.

Circadian oscillations of cytosine modifications may be confounded by cyclic influx of white blood cells into solid tissues $^{19,20}$. In such cases, changes in the proportions of cells may mimic osc-modCs due to contrasting epigenomes between penetrating blood cells and native tissue cells. If cell counts are a confounder, cell-specific non-circadian mRNAs should exhibit evidence for circadian oscillations. We investigated two noncircadian mRNAs for each of hepatocytes, pneumocytes, and macrophages. None of these cell-specific transcripts showed significant circadian oscillations (Supplementary Fig. 6 and Supplementary Table 2), suggesting that osc-modC were not simulated by changing cell counts.

Osc-modCs in circadian genes and E-box motifs. To test the functional association between osc-modCs and mRNA levels, we compared tissue-matched public circadian transcriptomic data sets $^{f}$ ( $n=72$ for both the liver and lung) with cytosine modification oscillations detected in each tissue. The oscillations for a given gene were summarized as the median coefficient of 

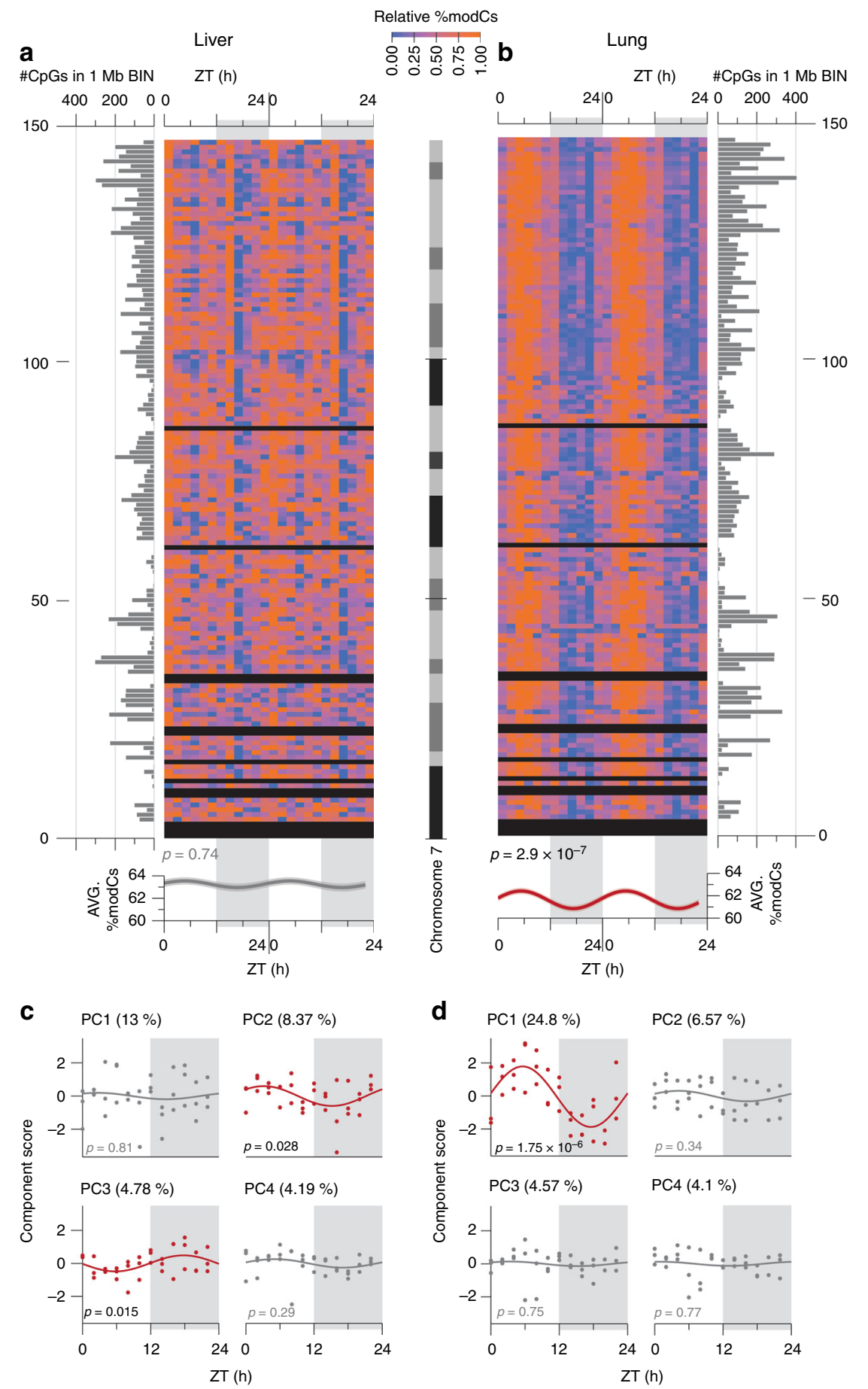

Fig. 3 Global patterns of oscillations in the 9-mo mouse liver and lung. $\mathbf{a}$, $\mathbf{b}$ A heatmap of the mean modification densities of epigenetically variable cytosines (EVCs) across chromosome 7 of $\mathbf{a}$ the liver and $\mathbf{b}$ the lung, normalized to a range of 0-1 in each $1 \mathrm{Mb}$ bin. The horizontal bar plots display the number of EVCs in each bin (bins with no EVCs appear black in the heatmap). The plots in the bottom panel display the chromosome-wide means of cytosine modification as a function of ZT, fitted using the harmonic regression model. The shading around the regression lines represents the $95 \%$ confidence band. All data were double plotted to aid with visualization. ZT, Zeitgeber time; modC, cytosine modification

determination (i.e., $R^{2}$ of the harmonic regression fit) of all EVCs within that gene. Our observations were threefold.

First, mRNA levels positively correlated with their corresponding cytosine modification oscillations in liver and lung tissues (weighted Pearson's $r=0.14$ and $0.19 ; p=4.1 \times 10^{-6}$ and $1.4 \times 10^{-10}$, respectively), suggesting that genes with more robust oscillations tend to be more abundantly expressed (Supplementary Data 1213). Second, circadian oscillations of mRNA were positively correlated with oscillations of their corresponding gene modifications (weighted Pearson's $r=0.075$ and $0.19 ; p=0.015$ and $4.3 \times$ $10^{-10}$, for the liver and lung, respectively), indicating that circadian transcripts tend to oscillate together with osc-modCs in their gene body. Third, acrophases of mRNAs were shifted by 0-6 h (range of significant phase shifts with Bonferroni corrected permuted $p<0.05$ ) from the nadir (ZT when oscillation reaches its minimum) of their matched, modC densities (Fig. 5a-b and 

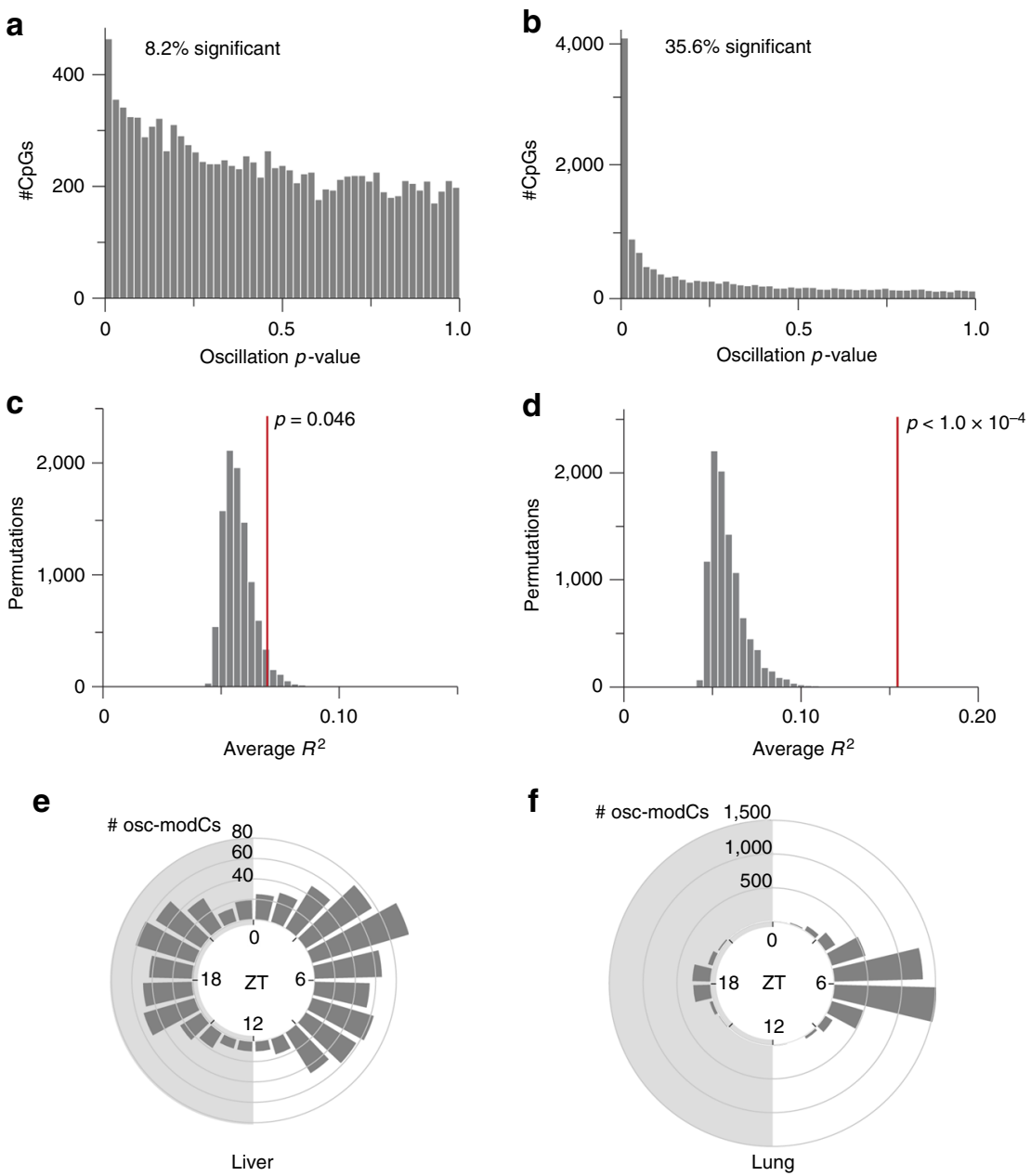

Fig. 4 Characterization of osc-modCs in the 9-mo mouse liver and lung. $\mathbf{a}, \mathbf{b}$ The $p$-value histogram of harmonic regression fits on individual cytosines showing that $8.2 \%(983$ of 11,941$)$ and $35.6 \%(5,054$ of 14,199$)$ of EVCs were oscillating $(p<0.05)$ in a the liver and $\mathbf{b}$ the lung, respectively. $\mathbf{c}, \mathbf{d}$ Average proportion of variance explained $\left(R^{2}\right)$ by the harmonic regression fits across all EVCs in each of 10,000 permutations of ZT labels. The red line depicts the observed average $R^{2}$ in $\mathbf{c}$ the liver and $\mathbf{d}$ the lung. e, $\mathbf{f}$ Acrophase rose plot showing modification peak times of osc-modCs in $\mathbf{e}$ the liver and in $\mathbf{f}$ the lung. osc-modC, oscillating cytosine modifications

Supplementary Fig. 7), suggesting a temporal relationship between the two events. As circadian mRNAs only partially reflect oscillations in nascent transcription ${ }^{11}$ and given the regulatory proximity of cytosine modification to nascent transcription, osc-modCs may be more robustly associated with oscillations in nascent transcription than mature mRNA.

Sequences flanking osc-modCs contained both canonical (CANNTG) and non-canonical (CANNNTG, GANNTG) E-box motifs $^{21}\left(e\right.$-value $=8.3 \times 10^{-26}-5.2 \times 10^{-212}$; Fig. $5 \mathrm{c}$-d and Supplementary Data 14-15). E-box response elements play key roles in regulation of circadian transcripts ${ }^{5,6}$. It has been shown that MycMAX heterodimer complex can interact with E-box motifs ${ }^{22}$ and Myc can in turn interact with DNA methyltransferase $3 \mathrm{~A}^{23}$ to methylate proximal CpG sites. In all, our findings show that oscmodCs are intricately linked to circadian transcriptomics.

Differential acrophase timing of osc-modCs. We categorized osc-modCs into "sleep acrophases" (ZT0-12) and "wake acrophases" (ZT12-24) (Fig. 3i-j), and observed that the acrophase time was associated with the osc-modC average modification density. In both liver and lung tissues, osc-modCs with wake acrophases showed a significantly higher average cytosine modification density (mean $\pm \mathrm{SE} ; 67 \pm 1.20 \%$ and $62 \pm 0.74 \%$, respectively) compared with osc-modCs with sleep acrophases
$(54 \pm 1.25 \%$ and $50 \pm 0.36 \%$, respectively). Modification densities of oscillating cytosines from the two acrophase peaks exhibited the largest modification differences during ZT12-24, whereas during ZT0-12 the densities were closest to each other (Fig. 5e-f). Borrowing from the field of astronomy, the cytosine modification densities were at their "apogee" at ZT18, as the distance between the two sinusoidal functions arrived at its maximum, and conversely reached their "perigee" at $\sim$ ZT6, as the distance between the two arrived at its minimum.

Osc-modCs' association with age-correlated changes. Although aging and the disruption of circadian processes are closely linked ${ }^{3,4}$, the molecular mechanisms of this association are not clear. It is conceivable that DNA modification can provide the platform to mediate this association due to its role in both the aging and circadian processes. Therefore, we investigated whether osc-modCs are related to age-correlated cytosine modifications (age-modCs).

We used a linear regression model to detect cytosine modification changes that either increased or decreased with age in the 9-, 15-, and 25-mo mice. We found that liver samples had more age-modCs compared with the lung $(24.1 \%$ and $8.4 \%$ of EVCs, with $p<0.05$ after Bonferroni correction, in the liver and lung, respectively; Supplementary Fig. 8 and Supplementary 


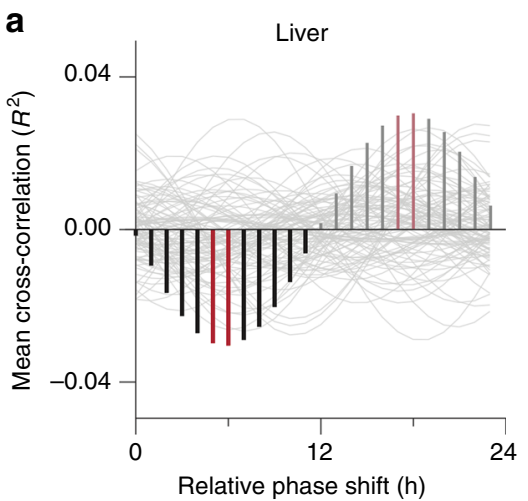

C

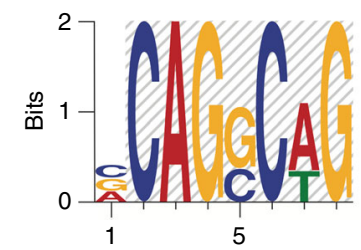

e

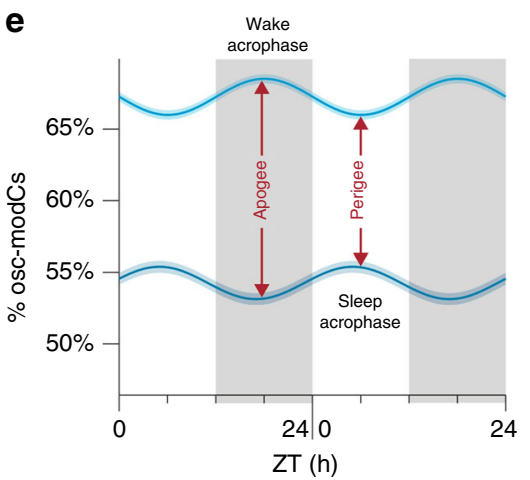

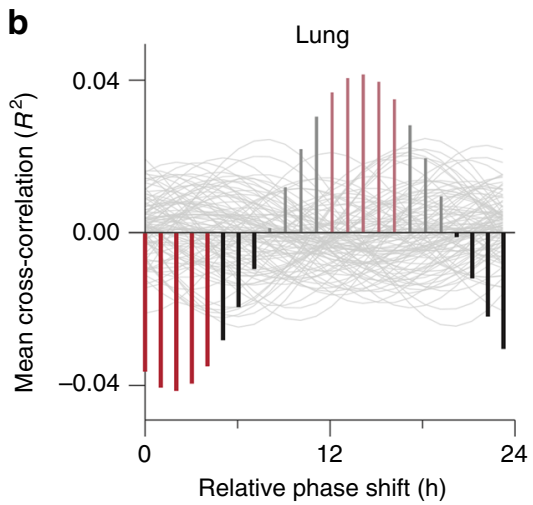

d

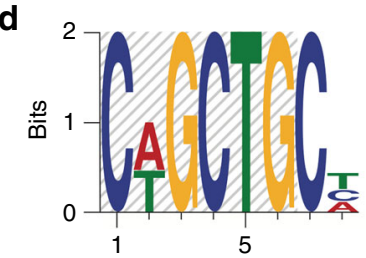

$\mathbf{f}$

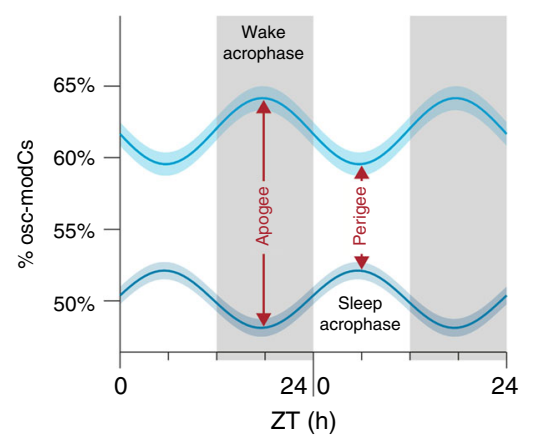

Fig. 5 The transcriptional and temporal coordination of oscillating modified cytosines in the 9-mo mouse liver and lung. a, b Mean gene-body modification densities of epigenetically variable cytosines in $\mathbf{a}$ the liver and $\mathbf{b}$ the lung were cross-correlated with corresponding circadian mRNA profiles after each iteration of a forward $1 \mathrm{~h}$ phase shift in the mRNA profiles. Bars represent the observed mean cross-correlation between mRNAs and modification densities for a given phase shift (red bars indicate Bonferroni corrected permuted $p<0.05$ ). The positive faded bars are mirror images of the negative bars (by nature of correlating phase shifted waves, a wave shifted by half a period anti-correlates with itself). Fine gray curves in the background depict crosscorrelations of 100 randomly selected (out of 10,000) permutations where both ZT labels and mRNA-gene modification density pairs were shuffled. c, $\mathbf{d}$ Representative E-box motifs enriched within $100 \mathrm{bp}$ in either direction of osc-modCs in $\mathbf{c}$ the mouse liver and $\mathbf{d}$ the lung. e, $\mathbf{f}$ Harmonic regression fits on the average modification density of osc-modCs with wake and sleep acrophases in $\mathbf{e}$ the mouse liver and $\mathbf{f}$ the lung, as a function of ZT. Shading around the regression lines represents the $95 \%$ confidence band. $\mathrm{ZT}$, Zeitgeber time; modC, cytosine modification

Data 16-17). Osc-modCs from the 9-mo mice showed a strong association with age-modCs in both the liver and lung tissues $\left(\mathrm{OR}=2.3(2.0-2.7)\right.$ and $1.4(1.2-1.6) ; p=2.6 \times 10^{-24}$ and $4.0 \times 10^{-5}$, respectively). In addition, the circadian amplitudes were correlated with the magnitude of the epigenetic aging effects and accounted for $18.4 \%$ (Pearson's $r=0.43 ; p=1.3 \times 10^{-14}$ ) and $72.8 \%$ (Pearson's $r=0.85 ; p=3.4 \times 10^{-111}$ ) of the age-dependent variance, in the liver and lung, respectively (Fig. 6a-b).

The associations between sleep or wake acrophases and agedependent gain or loss of cytosine modification were highly asymmetric; cytosines with sleep acrophases that exhibited an aging effect showed increased modification with age, whereas those with wake acrophases predominantly lost modification with age $\left(\mathrm{OR}=68(30-166)\right.$ and $394(113-1,875) ; p=1.4 \times 10^{-44}$ and $9.6 \times 10^{-46}$, in liver and lung tissues, respectively; Fig. $\left.6 \mathrm{c}-\mathrm{f}\right)$. The association between osc-modC acrophase time and direction of change in age-modC may provide insights into why genomic elements that are polarized in terms of DNA modification densities converge toward the mean in the aging epigenome ${ }^{24,25}$.
If osc-modCs have a causative influence on age-dependent epigenetic changes, the former would precede the latter chronologically. Therefore, osc-modCs that are exclusively present in younger animals (i.e., 9-mo) should be enriched for cytosines whose modifications show an aging trend after 9 months. Conversely, if aging induces osc-modCs, age-modCs should become more abundant amongst the osc-modCs specific to the older groups (i.e., 15- and 25-mo). Consistent with a circadian causative direction, cytosines oscillating only in the 9mo showed an enrichment of age-modCs (binomial $p=3.5 \times 10^{-8}$; $12.4 \%$ age-modCs), whereas cytosines oscillating only in the 15 mo or only in the 25-mo showed no enrichment or even depletion of age-modCs (binomial $p=0.42$ and $0.0018 ; 7.6 \%$ and $4.3 \%$ age-modCs, respectively; Fig. $6 \mathrm{~g}$ ). Interestingly, osc-modCs common to all three age groups also showed a significant depletion of age-modCs (binomial $p=0.0057 ; 5.3 \%$ age-modCs), suggesting that the proposed circadian-aging conversion has not occurred yet but may take place in the animals living longer than 25 months. We repeated this analysis using matched sample sizes or matched proportions of osc-modCs across all age groups and 
a

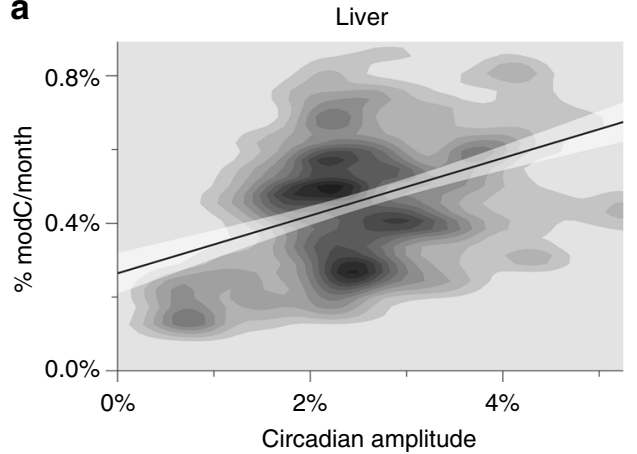

C
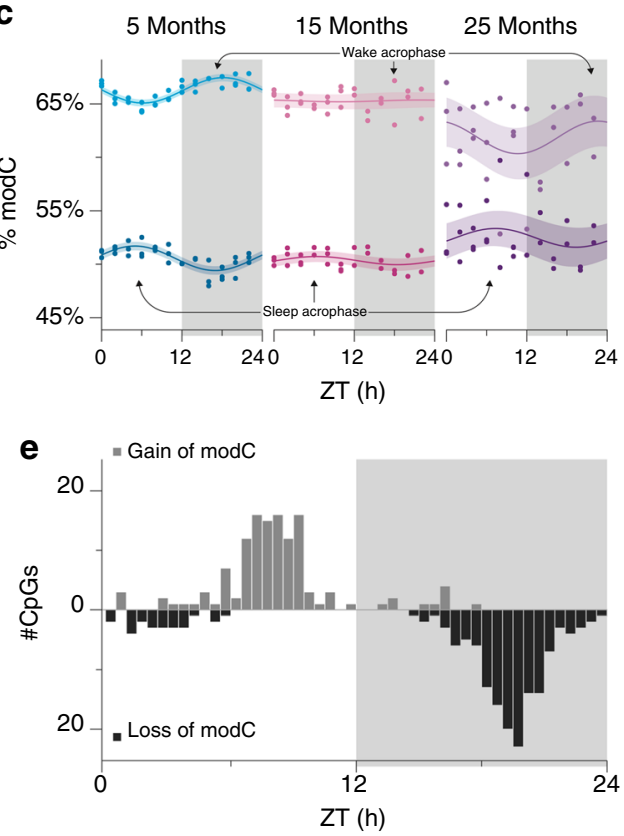

b

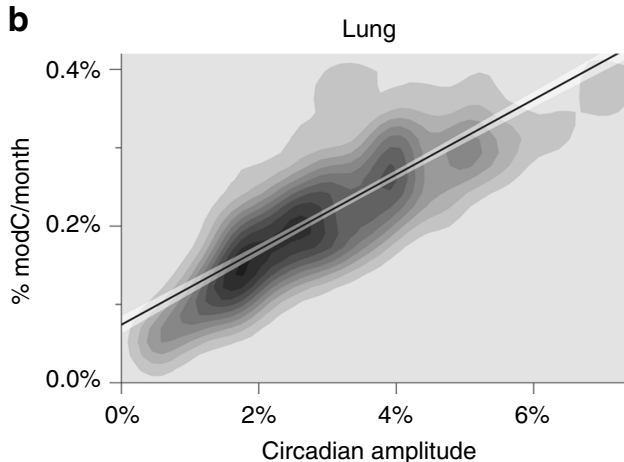

d

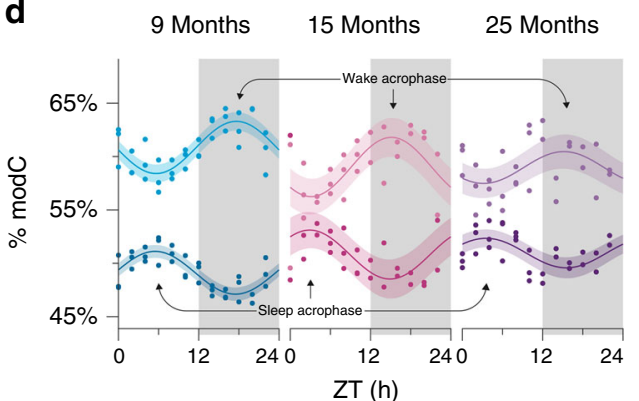

$\mathbf{f}$

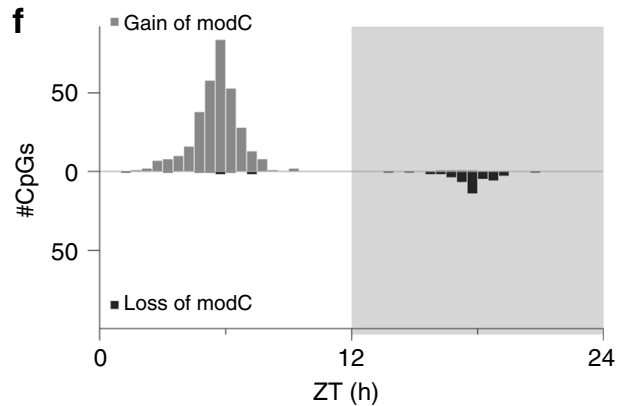

g
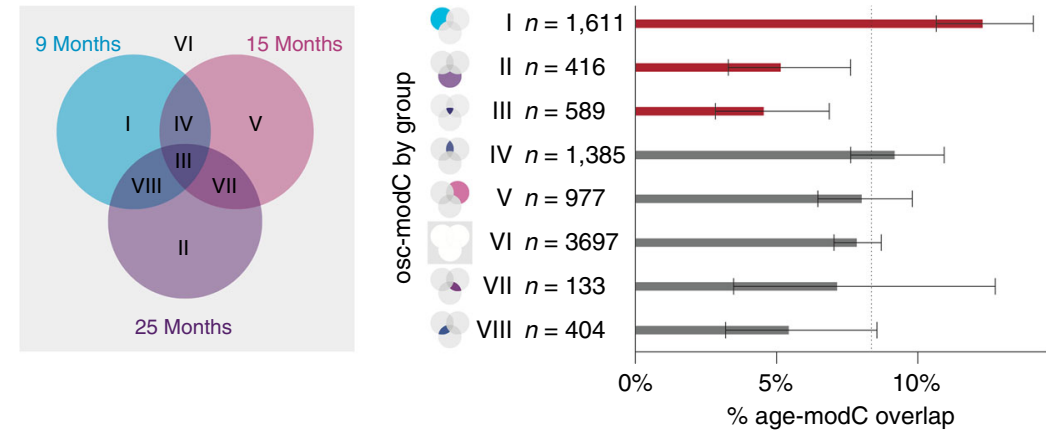

Fig. 6 The relationship between oscillating and age-correlated cytosine modifications in the mouse liver and lung. $\mathbf{a}$, $\mathbf{b}$ Density contour plots of the agemodC magnitude and the osc-modC amplitude in $\mathbf{a}$ the liver and $\mathbf{b}$ the lung. Contours were rendered using 2D kernel density estimation and the lines represent linear regression fits. Shading around the regression lines represents the $95 \%$ confidence band. $\mathbf{c}$, $\mathbf{d}$ The mean modification levels of 9 -mo oscmodCs, stratified by wake and sleep acrophases, converge with increasing age in the $\mathbf{c}$ the liver and $\mathbf{d}$ the lung. The curves show harmonic regression fits of the mean cytosine modification and the shading around the regression lines show $95 \%$ confidence bands. $\mathbf{e}, \mathbf{f}$ The relationship between acrophase time and aging direction in $\mathbf{e}$ the liver and $\mathbf{f}$ the lung. The white areas represent periods of lights on (i.e., sleep), whereas the shaded areas show periods of lights off (i.e., wake). The gray bars show counts of cytosines with gain of modification with age, whereas the black bars show counts for loss of modification. $\mathbf{g}$ OscmodC were categorized according to their placement in the Venn diagram. The bar plots represent the percentage of cytosines in each group that were also age-modCs with 95\% confidence intervals. The dashed line represents the expected proportion of age-modC and the red bars show significant $(p<$ 0.05) deviation from this expected line. Age-modC, age-correlated cytosine modifications; Osc-modC, oscillating modified cytosines; modC, modified cytosines; ZT, Zeitgeber time 
arrived at the same conclusions (Supplementary Fig. 9). These findings suggest that oscillations of cytosine modification could precede age-correlated cytosine modification changes, but additional experiments are required to establish causality.

As epigenetic factors are intimately related to transcription, we asked whether associations between circadian rhythmicity and aging also extend to the transcriptome. We compared publicly available circadian ${ }^{7}$ ( $n=72$ for liver and lung) and aging ${ }^{26,27}(n=$ 7 and 9 for liver and lung, respectively) transcriptomes (Supplementary Data 18-19) of mouse tissues and detected a significant overlap between the two $(\mathrm{OR}=1.3(1.2-1.4)$ and 1.6 (1.5-1.8); $p=1.3 \times 10^{-8}$ and $1.3 \times 10^{-25}$ for the liver and lung, respectively). We also found that amplitudes of oscillating transcripts correlate with magnitudes of aging effects $(r=0.65$ and $0.56 ; p=4.4 \times 10^{-170}$ and $9.4 \times 10^{-83}$ in the liver and lung, respectively) (Supplementary Fig. 10a-b). Gene Ontology (GO) analysis of the mRNAs common to both data sets showed enrichment of various terms including catabolic and metabolic processes in the liver (false discovery rate (FDR) $q=0.05-8.9 \times 10^{-24}$; Supplementary Fig. 10c and Supplementary Data 20), and cell adhesion and migration in the lung (FDR $q=0.05-1.5 \times 10^{-6}$; Supplementary Fig. 10d and Supplementary Data 21). These findings suggest that the circadian-aging association could be universal and therefore also detectable in metabolomic and proteomic studies.

\section{Discussion}

The evidence for daily cyclical patterns of cytosine modifications reported in this study makes cytosine modification a bona fide member of the cellular circadian machinery alongside oscillating RNAs, histone modifications, proteins, and metabolites. The underpinnings of the circadian regulation include numerous interdependent feedback loops, which precludes a clear demarcation of the hierarchy of such elements. Consideration of the temporal dimension is necessary for unravelling the complex molecular circuitries of the cell.

Circadian patterns of cytosine modification may help elucidate several poorly understood epigenetic phenomena. Osc-modCs can, in part, explain the occurrence of ongoing active demethylation and production of $\mathrm{hmC}$ in differentiated somatic cells.

Osc-modCs can explain a large fraction of variation in cytosine modifications and therefore this work indicates that circadian mismatched samples could result in findings that are confounded by oscillations. Failure to perform experiments in a circadianinformed manner may conflate epigenetic (alongside transcriptomic, metabolomic, and proteomic) differences arising from circadian mismatches with those attributed to underlying traits.

Epigenetic variation due to cytosine oscillation may even be larger than our estimates. In our study, we captured the composite effect of oscillations that occur in a large population of cells. Individual cells may exhibit differences with respect to the specific cytosine positions that undergo circadian oscillations within a genomic region. These subtle intercellular variations in daily reprogramming can create epigenetic heterogeneity within a specific region or genomic element. This could be a potential mechanism generating intermediate cytosine modifications in regulatory elements such as DNase hypersensitive sites and enhancers ${ }^{28}$.

We observed similarities and distinct differences in aging and osc-modC profiles between the mouse liver and lung tissues, namely a smaller number of osc-modCs but a larger number of age-modCs in the liver. It is possible that a faster rate of aging in liver can lead to diminished oscillations and this difference in signal-to-noise ratio would reduce the robustness of the oscmodC/age-modC association in the liver.
At present, opinions regarding the contribution of circadian dysfunction to aging differ significantly ${ }^{3,29}$. We detected that oscmCs are strongly associated with age-dependent changes in the epigenome and attempted to uncover the direction of such association. To address the relationship between the two epigenomic phenomena, Bradford Hill's causality criteria ${ }^{30}$ may be used. Four of the criteria supported a causal role for osc-modCs in aging by a varying extent: (I) circadian oscillation of cytosine modifications preceded age-modC (temporality); (II) amplitudes of circadian oscillation correlated with the size of the aging effect (gradient); (III) both cytosine modification and transcriptomes showed robust circadian-aging associations (consistency); and (IV) it is conceivable that daily epigenetic reprogramming errors gradually accrue over time converting the circadian epigenome into the aging epigenome (plausibility).

The circadian DNA modification may shed a new light on the associations between aging epigenomes and complex diseases, especially the ones with late age of onset. Deterministic, and not stochastic, accumulation or loss of modCs during the lifetime of an individual explains why DNA modification markers can be precise predictors of biological age ${ }^{31}$. Accelerated epigenetic aging, which is associated with increased morbidity and mortality $^{32-34}$, may be a result of deviated trajectories of circadian DNA modification. Our finding of the circadian origin of epigenetic convergence is relevant not only to aging but also to carcinogenesis; it suggests a common mechanism between agedependent DNA modification changes and cancer epigenome ${ }^{35}$. Hypothetically, even a small (e.g., 0.1\%) unidirectional daily asymmetry in circadian DNA demethylation-remethylation cycle can result in a bona fide epigenomic state of a malignancy in several months.

The circadian clock evolved before the advent of air travel, light bulbs, and constant food availability; it does not contain the necessary counterbalances for these stressors ${ }^{36}$. The plasticity and programmability of cytosine modification and the circadian clock make them amenable to continuous environmental inputs that can ultimately facilitate both circadian dysregulation or the correction of such dysregulations. Circadian parameters can be altered by various factors, including diet and chemical compounds, and such circadian manipulations may have an impact on the molecular trajectories during aging. In other words, it may be possible to potentially influence aging outcomes by modifying circadian epigenomes and transcriptomes at a younger age.

\section{Methods}

Sample information. C57BL/6JRj male mice were obtained from Janvier Labs. The animals were singly housed in polypropylene cages $(44 \times 22 \times 19 \mathrm{~cm})$ to facilitate assessment of activity. They were given ad libitum access to food, water, and a 17 $\mathrm{cm}$ diameter running wheel mounted in the cage. The animals were entrained to a $24 \mathrm{~h}$ light-dark cycle maintained at $12 \mathrm{~h}$ light and $12 \mathrm{~h}$ dark, where ZT0 refers to the light onset, for a minimum of 30 days. Wheel running activity was monitored using Vitalview (Phillips-Respironics) and circadian entrainment (i.e., wheel running activity onset) was verified using Actiview (Phillips-Respironics). At ages 9 , 15 , or 25 months (mo), mice were killed by cervical dislocation every $2 \mathrm{~h}$ over the course of $58 \mathrm{~h}$ (minimum). Collected tissues were snap frozen in liquid nitrogen and stored at $-80^{\circ} \mathrm{C}$.

Ethical approval. All experiments were approved by the Centre for Addiction and Mental Health Research Ethics Board (protocol 567), the University of Toronto Animal Care Committee (protocol 20010315), and performed in accordance with relevant guidelines and regulations.

DNA and RNA extraction. Frozen mouse tissues were digested in lysis buffer (35 mM EDTA, $75 \mathrm{mM} \mathrm{NaCl}, 10 \mathrm{mM}$ Tris- $\mathrm{HCl} \mathrm{pH}$ 8.0, $1 \%$ SDS, and $2 \mathrm{mg} \mathrm{ml}^{-1}$ Proteinase K) overnight and extracted using the phenol : chloroform method. Genomic DNA quality and quantity were examined on a $1 \%$ agarose gel, a NanoDrop 2000 Spectrophotometer (Thermo Fisher Scientific), and a Qubit 2.0 fluorometer (Invitrogen). 
For RNA studies, frozen tissues were treated with RNAlater-ICE Frozen Tissue Transition Solution (Ambion) and total RNA was extracted with an RNeasy Mini Kit (Qiagen). Total RNA was quantified using a NanoDrop 2000 Spectrophotometer (Thermo Fisher Scientific) and an Agilent 2100 Bioanalyzer with a RNA 6000 Nano Kit (Agilent). The investigated RNA samples had minimum RNA Integrity Number of 7.5.

Bisulfite and oxBS conversion. A total of $750 \mathrm{ng}$ of genomic DNA was bisulfite converted using an EZ DNA Methylation Kit (Zymo) according to the manufacturer's protocol for the HumanMethylation450 BeadChip (Illumina), with the following modifications suggested by the manufacturer for a more stringent conversion: $7.5 \mu \mathrm{l}$ of M-dilution buffer was used for the reaction, which was incubated at $42{ }^{\circ} \mathrm{C}$ for $30 \mathrm{~min}$ before addition of the CT-Conversion Reagent. A total of 185 $\mu \mathrm{L}$ of the M-dilution buffer was used in the preparation of the CT-Conversion Reagent, and only $97.5 \mu \mathrm{lL}$ of the reagent was added per reaction. oxBS conversion was performed using a TrueMethyl kit (CEGX) as per the manufacturer's recommendations. Using $\mathrm{CpH}$ methylation as a proxy for bisulfite conversion efficiency, the mean non-conversion rate for all experiments were estimated at 0.6 $\pm 0.6 \%($ mean $\pm \mathrm{SD})$.

Bisulfite padlock probe and library preparation. Bisulfite padlock probes were designed using the ppDesigner 2.0 software $^{16}$. The reference mouse genome (mm10) was masked for genomic variations and repeats (dbSNP 138, Microsatellites, RepeatMasker, Segmental Dups, Simple Repeats, and WindowMasker + SDust). For the remaining (non-repetitive) chr 7 sequence, all possible probe arms were designed and filtered for the presence of at least one $\mathrm{CpG}$ and less than $10 \%$ of the masked genomic sequence within the targeted region. Seven basepair unique molecular identifiers (UMIs) were added to the common sequence of each probe immediately adjacent to the probe annealing arms and later used for removal of PCR duplicates. The probes were printed by CustomArray (Bothell, WA, USA) and prepared according to the published protocol ${ }^{16}$ with minor modifications.

Briefly, $1-100 \mathrm{nM}$ of the synthesized probes were amplified using $400 \mathrm{nM}$ of pAP1V61U (5'-G* $G^{*} G$ TCATATCGGTCACTGTU-3') and AP2V6 (5'-5PhosCACGGGTAGTGTGTATCCTG- $3^{\prime}$ ) primers, and $1 \times$ KAPA SYBR Fast $\mathrm{qPCR}$ mix in four separate $50 \mu \mathrm{l}$ reactions. The following cycling conditions were used: $95^{\circ} \mathrm{C}$ for $30 \mathrm{~s}, 15$ cycles of $95^{\circ} \mathrm{C}$ for $10 \mathrm{~s}, 55^{\circ} \mathrm{C}$ for $20 \mathrm{~s}$, and $70^{\circ} \mathrm{C}$ for $30 \mathrm{~s}$, with a final extension of $70^{\circ} \mathrm{C}$ for $2 \mathrm{~min}$. The amplicons were pooled and purified using QIAquick PCR purification kit (Qiagen) following the manufacturer's recommendation. The final product $(0.2 \mathrm{nM})$ was used as template for a large-scale production PCR involving a minimum of four 96-well plate reactions amplified in identical conditions to the first round. The amplicons were then pooled and concentrated using ethanol precipitation. The amplicons were re-purified using QIAquick PCR purification kit (Qiagen) following the manufacturer's recommendation. Amplification adaptors were then removed using three enzymatic digestions. First, $15-20 \mu \mathrm{g}$ of the purified amplicon were mixed with 50 units of lambda exonuclease (New England Biolabs) in a $150 \mu$ reaction containing $1 \times$ lambda exonuclease buffer and incubated for $1 \mathrm{~h}$ at $37^{\circ} \mathrm{C}$, to remove the bottom strand. The digested amplicons were purified using ssDNA/RNA clean \& concentrator kit (Zymo) using the manufacturer's protocol. Second, 3-5 $\mu \mathrm{g}$ of the single-stranded probes were then digested with 5 units of USER (New England Biolabs) in an $80 \mu \mathrm{l}$ reaction containing $1 \times$ DpnII buffer (New England Biolabs) and incubated for $1 \mathrm{~h}$ at $37^{\circ} \mathrm{C}$. Next, $5 \mu \mathrm{l}$ of $100 \mu \mathrm{M}$ guide oligo $\left(5^{\prime}\right.$ -

GTGTATCCTGATC- $3^{\prime}$ ), $2 \mu \mathrm{l}$ of $10 \times$ DpnII buffer and $8 \mu$ of water were added to the mix and the reaction was incubated at $94^{\circ} \mathrm{C}$ for $2 \mathrm{~min}$, followed by $3 \mathrm{~min}$ at $37^{\circ} \mathrm{C}$. Third, 250 units of DpnII (New England Biolabs) were added and the reaction was incubated at $37^{\circ} \mathrm{C}$ for $2 \mathrm{~h}$ followed by heat inactivation at $75^{\circ} \mathrm{C}$ for $20 \mathrm{~min}$. The probes were then purified using a TBE-Urea denaturing gel and cutting the band corresponding to $\sim 120 \mathrm{bp}$.

For the padlock library preparation, $1.5 \mathrm{ng}$ of the purified probes was mixed with $200 \mathrm{ng}$ bisulfite-treated genomic DNA (quantified using Qubit ssDNA Assay Kit) in a $20 \mu \mathrm{l}$ reaction containing $1 \times$ ampligase buffer and covered with $20 \mu \mathrm{l}$ of mineral oil to prevent evaporation. The reaction was then incubated at $94^{\circ} \mathrm{C}$ for 30 $\mathrm{s}$ and gradually lowered $\left(-0.5^{\circ} \mathrm{C} / 25 \mathrm{~s}\right)$ to $55^{\circ} \mathrm{C}$ and incubated for an additional 20 $\mathrm{h}$. With the plate still in the thermocycler, a $6.5 \mu \mathrm{l}$ mixture containing $2.5 \mu \mathrm{l} 10 \times$ $\mathrm{NAD}^{+}(\mathrm{NEB}), 0.85 \mu \mathrm{dNTP}(1 \mathrm{mM}), 0.85 \mu \mathrm{l}$ ampligase (Epicentre, Madison, Wisconsin, USA), $0.85 \mu \mathrm{l} 10 \times$ ampligase buffer, and $1.5 \mu \mathrm{l}$ of preheated PfuTurbo Cx Hotstart DNA Polymerase (Agilent Genomics) was added. The unligated products were removed using 20 units of exonuclease I and 200 units of exonuclease III (Epicentre). The circularized DNA was then enriched by PCR using $5 \mu \mathrm{l}$ of the reaction in a $50 \mu \mathrm{l}$ volume containing $200 \mathrm{nM}$ of Amp_F_SE and SE_Amp_IndX indexing primers (Supplementary Data 22) and $1 \times$ KAPA SYBR Fast qPCR master mix using a StepOnePlus Real-Time PCR System (Thermo Fisher Scientific) at the following cycling conditions: $95^{\circ} \mathrm{C}(30 \mathrm{~s}) ; 8$ cycles of $95^{\circ} \mathrm{C}$ $(10 \mathrm{~s}), 58^{\circ} \mathrm{C}(30 \mathrm{~s})$, and $72^{\circ} \mathrm{C}(20 \mathrm{~s}) ; 15$ cycles of $95^{\circ} \mathrm{C}(10 \mathrm{~s})$ and $72^{\circ} \mathrm{C}(20 \mathrm{~s})$; and a final extension at $72^{\circ} \mathrm{C}(3 \mathrm{~min})$. The PCR products were purified using $0.7 \times$ volume of AMPure magnetic beads (Beckman Coulter) with two $70 \%$ ethanol washes, and quantified using a Qubit dsDNA HS assay (Thermo Fisher Scientific). Equal amounts of each sample were pooled, and the band at $\sim 360$ bp was excised and purified using standard agarose gel extraction methods. The purified libraries were quantified for sequencing using KAPA Library Quantification kits.
Preprocessing of sequencing data. The libraries were sequenced on a HiSeq 2500 platform (Illumina) at $2 \times 125$ paired-end reads by using custom sequencing primers (Supplementary Data 22). For each FASTQ file, the UMIs were removed from the start of the reads and saved for later processing. The FASTQ reads were quality trimmed using Trimmomatic ${ }^{37}$ for trailing bases with a phred score $<30$ and all reads with post-trimming length $<50 \mathrm{bp}$. The trimmed reads were then aligned to a masked genome (mapping only to regions within a $100 \mathrm{bp}$ window of the known probe locations) using Bismark v0.14.3 ${ }^{38}$ and Bowtie 2 v2.2.2 $2^{39}$. The aligned reads were further filtered to only include reads that had start and end positions matching the padlock probe annealing arm sequence with no more than one mismatch. The filtered reads were subsequently PCR de-duplicated using the UMIs. The filtered read pairs were then converted to modification calls using the Bismark methylation extractor tool ${ }^{38}$.

Samples with low coverage were removed based on a comparison relative to other samples within the same experiment. Individual CpGs were required to have a minimum coverage of 30 reads in each sample for inclusion in the analysis. $\beta$ Values were calculated as the proportion of cytosines and thymines for a given $\mathrm{CpG} ; \beta=\mathrm{M} /(\mathrm{M}+\mathrm{U})$, where $\mathrm{M}=$ number of cytosines (modCs) and $\mathrm{U}=$ number of thymines (unmodified cytosines). All samples for a given age and tissue were internally correlated to identify outliers, and samples with average inter-sample correlation value more than $3 \mathrm{SD}$ below the mean were excluded from further analysis.

$\mathrm{hmC}$ data were derived from the set of cytosines which were epigenetically variable (see "Detection of EVC in mouse tissues") in either $\mathrm{mC}$ (from oxBS padlock sequencing) or modC (from bisulfite padlock sequencing) data sets. $\mathrm{hmC}$ values were estimated by subtracting the oxBS sequencing densities from the bisulfite sequencing densities following the TrueMethyl kit (Cambridge Epigenetix, Cambridge, UK) recommendations.

Lung $\mathrm{mC}$ data were obtained from a larger set of probes that, in addition to chromosome 7, targeted other chromosomes. Only probes overlapping with the primary chromosome 7 probe list were used for analysis. Outlier samples were calculated and removed prior to subsetting.

Detection of EVCs. To detect EVCs, whose biological signal exceeded technical variation, we analyzed three technical replicates for each biological sample. A oneway analysis of variance test comparing the variance between technical and biological replicates was performed on every cytosine. Each tissue and age groups were analyzed separately and cytosines with $p<0.05$ were identified as EVCs. Following EVC identification, the cytosine modification values of the three technical replicates were averaged using the median, in order to obtain a single robust biological data point for further analyses.

Detection of circadian oscillations. A harmonic linear regression model was used to identify circadian oscillations. The period was fixed to $24 \mathrm{~h}$, and the phase and the amplitude were modeled as a linear combination of sine and cosine terms as follows:

$$
y=b_{0}+b_{1} \sin \left(\frac{2 \pi \mathrm{ZT}}{24}\right)+b_{2} \cos \left(\frac{2 \pi \mathrm{ZT}}{24}\right)+\epsilon
$$

where $y$ is the observed modification level, $b_{0-2}$ are regression coefficients, ZT is the time of observation, and $\varepsilon$ is the error term. $P$-values were obtained by comparing this model to the null model without the sine and cosine terms using an F-test. Cytosines with harmonic regression $p<0.05$ were identified as osc-modCs with their amplitude $(A)$ and acrophase $(\phi)$ defined as:

$$
A=2 \sqrt{b_{1}^{2}+b_{2}^{2}}
$$

$$
\phi=\frac{12}{\pi} \operatorname{atan} 2\left(b_{1}, b_{2}\right) \bmod 24
$$

To determine whether the average proportion of variance explained by oscillations is higher than expected by chance, 10,000 permutations were performed by shuffling ZT labels and the mean $R^{2}$ value was calculated across the EVCs in each permutation. The permutation $p$-value was derived as a fraction of permutations with the permuted mean $R^{2}$ value greater than the observed.

PCA was used to quantify the amount of variability explained by oscillations. Principal components were calculated via singular value decomposition of the mean centered data matrix. The resulting scores of four main principal components were inspected for oscillations by fitting the harmonic regression model described above.

Analysis of circadian and tissue-specific transcripts. mRNAs of two key circadian genes, period circadian clock 2 (Per2) and aryl hydrocarbon receptor nuclear translocator like (Arntl), were used to confirm the circadian entrainment of mice ${ }^{1}$. Liver-, lung-, and macrophage-specific mRNA targets were selected using a public microarray expression data set (Gene Expression Omnibus (GEO) accession 
GSE1 $133^{40}$ ). The data were queried for genes whose expression exhibited the highest fold change between the tissue of interest and other tissues. Absence of circadian variation in the mRNA transcripts specific to hepatocytes, pneumocytes, and macrophages was verified using the CircaDB database ${ }^{41}$

Quantification of relative mRNA levels was performed on an Applied Biosystems ViiA 7 Real-Time PCR System (Applied Biosystems). First-strand complementary DNA synthesis was performed with a SuperScript III First-Strand Synthesis System (Thermo Fisher Scientific) on DNase I-treated RNA. Real-time PCR was performed in triplicate using Power SYBR Green PCR Master Mix (Applied Biosystems), with $400 \mathrm{nM}$ primers (Supplementary Table 3) in a total reaction volume of $10 \mu \mathrm{l}$ and at the thermal cycling conditions recommended by the manufacturer. Glyceraldehyde-3-phosphate dehydrogenase (Gapdh) mRNA levels were used as endogenous controls. The results were analyzed using ViiA 7 software and $\mathrm{R}^{42}$.

Analysis of the transcriptomic datasets. Pre-normalized public circadian transcriptomic microarray (GEO accession: GSE54650 ${ }^{7}$ ) and aging transcriptomic data sets (GEO accession: GSE57809 ${ }^{26}$ and GSE6591 ${ }^{27}$ for the liver and lung, respectively) were used for detection of circadian (see "Detection and analysis of circadian epigenetic oscillations") and nominally significant aging mRNAs (see "Aging analysis for the mouse tissue DNA samples"). Data sets were matched by RefSeq ID or gene symbol and genes with missing mRNA data were removed.

GO enrichment analysis on the public transcriptomic dataset was performed using GREAT $3.0^{43}$. A hypergeometric test was performed using oscillating and aging transcripts as foreground and all transcripts as background. The resulting $p$ values were FDR adjusted and significance threshold was set as $q<0.05$. To reduce the dimensionality of the GO enrichment terms to the most informative ones, the list of enriched terms $(q<0.05)$ was submitted to REViGO ${ }^{44}$ and terms with similar gene lists were reduced by merging terms with dispensability score $<0.7$.

Pearson's correlations between circadian transcriptomic data and median $R^{2}$ of cytosine modification data were weighted by the number of cytosines within the gene.

Cytosine modification and circadian mRNA phase shift. Each mRNA transcript was paired with the corresponding EVCs within its gene body. For each transcript, the harmonic model estimate from mRNA was correlated with the mean cytosine modification values within the same gene (Pearson's correlation). The overall correlation was summarized as the mean correlation value across all mRNA transcripts. In order to estimate the strength of each phase shift between mRNA and modification, the above procedure was repeated 24 times, each time shifting the obtained harmonic model fit by $1 \mathrm{~h}$. The procedure for a single phase shift can be represented as:

$$
\begin{gathered}
y_{i p}=b_{0 i}+b_{1 i} \sin \left(\frac{(\mathrm{ZT}-p) 2 \pi}{24}\right)+b_{2 i} \cos \left(\frac{(\mathrm{ZT}-p) 2 \pi}{24}\right)+\epsilon_{i}, \\
r_{i p}=\operatorname{cor}\left(x_{i}, y_{i p}\right), \\
r_{p}=\frac{\sum_{i=1}^{N} r_{i p}}{N} .
\end{gathered}
$$

where $y_{i p}$ is a vector of mRNA estimates at phase shift $p$, for each time of observed cytosine modification, ZT, for gene $i . b_{0 \mathrm{i}-2 \mathrm{i}}$ are the mRNA harmonic regression coefficients and $\varepsilon_{i}$ is the error term. cor is the function for Pearson's correlation coefficient and $x_{i}$ is a vector of mean modification values. The summarized strength of the correlation for phase shift $p$ is estimated by $r_{p}$, the mean of all genes' Pearson's $r, r_{i p}$.

To generate a null distribution, permutations $(N=10,000)$ were performed by shuffling the ZT times and pairing of mRNA and modification values. Each permutation produced 24 overall correlation estimates (i.e., 10,000 values for each phase shift) and for each phase shift the permutation $p$-value was calculated as the fraction of permutations with overall correlation greater than the observed value. The permutation $p$-values were corrected for multiple testing using the Bonferroni procedure.

Aging analysis. Only cytosines that were epigenetically variable across all three age groups were considered for aging effects. Cytosines exhibiting age-dependent modification across the three age groups (9-, 15-, and 25-mo) were identified using an F-test between a null intercept-only model $\left(y_{\text {null }}\right)$ and a linear model using age as a predictor $\left(y_{\text {alternative }}\right)$ defined as:

$$
\begin{gathered}
y_{\text {null }}=b_{0}+\varepsilon, \\
y_{\text {alternative }}=b_{0}+b_{1} \text { age }+\varepsilon .
\end{gathered}
$$

Cytosines whose modification showed a significant correlation with age (Bonferroni corrected $p<0.05$ ) were called age-correlated cytosines (age-modC) and the slope of the regression line (coefficient $b_{1}$ in $y_{\text {alternative }}$ model) was used to determine the direction of change.

Motif analysis. Sequence motifs were examined at the oscillating cytosine position $\pm 100 \mathrm{bp}$. Overlapping $200 \mathrm{bp}$ regions (i.e. redundant sequences) were merged into one sequence. MEME suite $4.10 .2^{45}$ was used to identify overrepresented sequences using the following parameters: -dna, -mod anr, -maxsites 1000, -nmotifs 20 , -evt 1e-10, -revcomp, -maxsize 1,00,00,000.

Analysis of differences in acrophase timing. Absolute acrophase differences (minor arc length) between paired data sets (liver 9-mo modC and lung 9-mo modC; liver $\mathrm{hmC}$ and $\mathrm{mC}$; and lung $\mathrm{hmC}$ and $\mathrm{mC}$ ) were calculated for each cytosine and averaged by taking the median. Permuted values were calculated by randomly shuffling acrophase pairings of one data set relative to the other and again computing median absolute acrophase differences. $P$-value was measured as the number of permutations with a value greater than (or less than, depending on the alternative hypothesis) observed in the real data.

Circadian-aging association analysis. Associations between osc-modC and aging probes were estimated using two-sided Fisher's exact test. Only EVCs were used to compute the contingency table. All computational analyses were performed in $\mathrm{R}^{42}$ unless specified otherwise.

Data availability. Next-generation sequencing data that support the findings of this study have been deposited in the GEO with the accession code GSE83947.

Received: 3 August 2017 Accepted: 20 December 2017

Published online: 13 February 2018

\section{References}

1. Bell-Pedersen, D. et al. Circadian rhythms from multiple oscillators: lessons from diverse organisms. Nat. Rev. Genet. 6, 544-556 (2005).

2. Takahashi, J. S., Hong, H. K., Ko, C. H. \& McDearmon, E. L. The genetics of mammalian circadian order and disorder: implications for physiology and disease. Nat. Rev. Genet. 9, 764-775 (2008).

3. Kondratova, A. A. \& Kondratov, R. V. The circadian clock and pathology of the ageing brain. Nat. Rev. Neurosci. 13, 325-335 (2012).

4. Froy, O. Circadian rhythms, aging, and life span in mammals. Physiol. (Bethesda) 26, 225-235 (2011).

5. Ripperger, J. A. \& Schibler, U. Rhythmic CLOCK-BMAL1 binding to multiple E-box motifs drives circadian Dbp transcription and chromatin transitions. Nat. Genet. 38, 369-374 (2006).

6. Reppert, S. M. \& Weaver, D. R. Coordination of circadian timing in mammals. Nature 418, 935-941 (2002).

7. Zhang, R., Lahens, N. F., Ballance, H. I., Hughes, M. E. \& Hogenesch, J. B. A circadian gene expression atlas in mammals: implications for biology and medicine. Proc. Natl Acad. Sci. USA 111, 16219-16224 (2014).

8. Eckel-Mahan, K. L. et al. Reprogramming of the circadian clock by nutritional challenge. Cell 155, 1464-1478 (2013).

9. Duong, H. A., Robles, M. S., Knutti, D. \& Weitz, C. J. A molecular mechanism for circadian clock negative feedback. Science 332, 1436-1439 (2011).

10. Katada, S. \& Sassone-Corsi, P. The histone methyltransferase MLL1 permits the oscillation of circadian gene expression. Nat. Struct. Mol. Biol. 17, 1414-1421 (2010).

11. Koike, N. et al. Transcriptional architecture and chromatin landscape of the core circadian clock in mammals. Science 338, 349-354 (2012).

12. Xia, L. et al. Daily variation in global and local DNA methylation in mouse livers. PLoS ONE 10, e0118101 (2015).

13. Azzi, A. et al. Circadian behavior is light-reprogrammed by plastic DNA methylation. Nat. Neurosci. 17, 377-382 (2014).

14. Vollmers, C. et al. Circadian oscillations of protein-coding and regulatory RNAs in a highly dynamic mammalian liver epigenome. Cell. Metab. 16, 833-845 (2012).

15. Lim, A. S. et al. 24-hour rhythms of DNA methylation and their relation with rhythms of RNA expression in the human dorsolateral prefrontal cortex. PLoS Genet. 10, e1004792 (2014).

16. Diep, D. et al. Library-free methylation sequencing with bisulfite padlock probes. Nat. Methods 9, 270-272 (2012)

17. Tahiliani, M. et al. Conversion of 5-methylcytosine to 5hydroxymethylcytosine in mammalian DNA by MLL partner TET1. Science 324, 930-935 (2009).

18. Booth, M. J. et al. Quantitative sequencing of 5-methylcytosine and 5hydroxymethylcytosine at single-base resolution. Science 336, 934-937 (2012). 
19. Haspel, J. A. et al. Circadian rhythm reprogramming during lung inflammation. Nat. Commun. 5, 4753 (2014)

20. Scheiermann, C., Kunisaki, Y. \& Frenette, P. S. Circadian control of the immune system. Nat. Rev. Immunol. 13, 190-198 (2013).

21. Fernandez, P. C. et al. Genomic targets of the human c-Myc protein. Genes Dev. 17, 1115-1129 (2003)

22. Desbarats, L., Gaubatz, S. \& Eilers, M. Discrimination between different Ebox-binding proteins at an endogenous target gene of c-myc. Genes Dev. 10, 447-460 (1996).

23. Brenner, C. et al. Myc represses transcription through recruitment of DNA methyltransferase corepressor. EMBO J. 24, 336-346 (2005).

24. Teschendorff, A. E., West, J. \& Beck, S. Age-associated epigenetic drift: implications, and a case of epigenetic thrift? Hum. Mol. Genet. 22, R7-R15 (2013).

25. Oh, G. et al. Epigenetic assimilation in the aging human brain. Genome Biol. 17, 76 (2016).

26. Bochkis, I. M., Przybylski, D., Chen, J. \& Regev, A. Changes in nucleosome occupancy associated with metabolic alterations in aged mammalian liver. Cell Rep. 9, 996-1006 (2014).

27. Misra, V. et al. Global expression profiles from C57BL/6J and DBA/2J mouse lungs to determine aging-related genes. Physiol. Genom. 31, 429-440 (2007).

28. Elliott, G. et al. Intermediate DNA methylation is a conserved signature of genome regulation. Nat. Commun. 6, 6363 (2015).

29. Lopez-Otin, C., Blasco, M. A., Partridge, L., Serrano, M. \& Kroemer, G. The hallmarks of aging. Cell 153, 1194-1217 (2013).

30. Hill, A. B. The environment and disease: association or causation? Proc. R. Soc. Med. 58, 295-300 (1965).

31. Horvath, S. DNA methylation age of human tissues and cell types. Genome Biol. 14, R115 (2013).

32. Chen, B. H. et al. DNA methylation-based measures of biological age: metaanalysis predicting time to death. Aging (Albany, NY) 8, 1844-1865 (2016).

33. Horvath, S. et al. An epigenetic clock analysis of race/ethnicity, sex, and coronary heart disease. Genome Biol. 17, 171 (2016).

34. Horvath, S. et al. Huntington's disease accelerates epigenetic aging of human brain and disrupts DNA methylation levels. Aging (Albany, NY) 8, 1485-1512 (2016).

35. Cruickshanks, H. A. et al. Senescent cells harbour features of the cancer epigenome. Nat. Cell. Biol. 15, 1495-1506 (2013).

36. Bass, J. \& Lazar, M. A. Circadian time signatures of fitness and disease. Science 354, 994-999 (2016).

37. Bolger, A. M., Lohse, M. \& Usadel, B. Trimmomatic: a flexible trimmer for Illumina sequence data. Bioinformatics 30, 2114-2120 (2014).

38. Krueger, F. \& Andrews, S. R. Bismark: a flexible aligner and methylation caller for Bisulfite-Seq applications. Bioinformatics 27, 1571-1572 (2011).

39. Langmead, B. \& Salzberg, S. L. Fast gapped-read alignment with Bowtie 2. Nat. Methods 9, 357-359 (2012).

40. Su, A. I. et al. A gene atlas of the mouse and human protein-encoding transcriptomes. Proc. Natl Acad. Sci. USA 101, 6062-6067 (2004).

41. Pizarro, A., Hayer, K., Lahens, N. F. \& Hogenesch, J. B. CircaDB: a database of mammalian circadian gene expression profiles. Nucleic Acids Res. 41, D1009-D1013 (2013).

42. R core Team: A language and environment for statistical computing ( $\mathrm{R}$ Foundation for Statistical Computing, Vienna, Austria, 2016).

43. McLean, C. Y. et al. GREAT improves functional interpretation of cisregulatory regions. Nat. Biotechnol. 28, 495-501 (2010).
44. Supek, F., Bosnjak, M., Skunca, N. \& Smuc, T. REVIGO summarizes and visualizes long lists of gene ontology terms. PLoS ONE 6, e21800 (2011).

45. Bailey, T. L. et al. MEME SUITE: tools for motif discovery and searching. Nucleic Acids Res. 37, W202-W208 (2009).

\section{Acknowledgements}

We thank E. Ellison for consultation regarding blood cells in solid tissues, T. Khare for advice on bisulfite padlock sequencing experiments, A. Lim for advice on circadian analyses, A. Wong for help with animal studies, and A. Krisciunas and P. Gibas for discussions regarding data analysis. We thank I. Meirelles, A. Norwood, and J. Yao for their consultations and assistance in generating the figures. This work was supported in part by the Canadian Institutes for Health Research, the National Institute of Mental Health, Brain Canada, and the Krembil Foundation (A.P.). J.G. and K.K. were funded by a grant (MIP-043/2014) from the Research Council of Lithuania.

\section{Author contributions}

A.P. conceived the project. G.O., S.E., and A.P. developed the theoretical framework and designed the experiments. S.E., A.Z., A.N., G.O., V.L., P.J., E.S.O., R.H.J., and M.S. performed the wet lab experiments. T.C.S. and M.R.R. performed the entrainment and monitoring of mice. G.O., M.C., J.G., K.K., and A.P. were responsible for the data analysis, presentation, and interpretation of the results. D.E.G. and G.O. developed the algorithms to process the sequencing data. G.O. and A.P. wrote the manuscript, with input from all co-authors.

\section{Additional information}

Supplementary Information accompanies this paper at https://doi.org/10.1038/s41467018-03073-7.

Competing interests: The authors declare no competing financial interests.

Reprints and permission information is available online at http://npg.nature.com/ reprintsandpermissions/

Publisher's note: Springer Nature remains neutral with regard to jurisdictional claims in published maps and institutional affiliations.

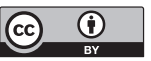

Open Access This article is licensed under a Creative Commons Attribution 4.0 International License, which permits use, sharing, adaptation, distribution and reproduction in any medium or format, as long as you give appropriate credit to the original author(s) and the source, provide a link to the Creative Commons license, and indicate if changes were made. The images or other third party material in this article are included in the article's Creative Commons license, unless indicated otherwise in a credit line to the material. If material is not included in the article's Creative Commons license and your intended use is not permitted by statutory regulation or exceeds the permitted use, you will need to obtain permission directly from the copyright holder. To view a copy of this license, visit http://creativecommons.org/ licenses/by/4.0/.

(C) The Author(s) 2018 